\title{
Spatial memory impairment in patients after tumour resection: evidence for a double dissociation
}

\author{
Roy P C Kessels, Albert Postma, L Jaap Kappelle, Edward H F de Haan
}

Psychological Laboratory, Utrecht University, The Netherlands

R P C Kessels

A Postma

E H F de Haan

Department of Neurology, University Medical Center Utrecht, The Netherlands L J Kappelle

Correspondence to: Mr R P C Kessels, Psychological Laboratory, Department of Psychonomics, Utrech University, Heidelberglaan 2, NL-3584 CS Utrecht, The Netherlands

r.kessels@fss.uu.nl

Received 17 December 1999 and in revised form 10 April 2000

Accepted 20 April 2000

\begin{abstract}
Human spatial memory can be divided into multiple, partly separate cognitive processes. In this study, object location memory was studied using a set of tasks that assessed three different spatial memory aspects: positional memory, object location binding, and a combined process. Also, maze learning and spatial span memory were measured. Ten patients who had undergone intracranial tumour resection participated, and their individual results were compared with control data from 24 healthy subjects. Four patients showed selective spatial memory impairments; two patients were impaired at positional memory, and two other patients were impaired on object location binding and the combined process. This double dissociation provides further evidence for the notion that object location memory is not a unitary system, but consists of at least two separate mechanisms. In addition, spatial memory problems were predominantly present in the patients with lesions in either the right hemisphere or in the parietal lobe. These results are in agreement with previous findings on the involvement of the right hemisphere and the posterior parietal cortices in spatial processing.

(F Neurol Neurosurg Psychiatry 2000;69:389-391)
\end{abstract}

Keywords: double dissociation; spatial memory; tumour resection

Spatial memory - that is, the ability to code, store, and retrieve information about the spatial layouts in our environment - enables us to learn a route or path between two points, or to remember the location of objects. Consequently, dysfunction in spatial memory can greatly impair daily life activities. ${ }^{1}$ Spatial memory is probably not a unitary construct, but might consist of multiple cognitive components. For example, a distinction can be made between allocentric spatial memory (memory for relative positions of stimuli, independent of the observer) and egocentric spatial memory (memory for positions of stimuli in relation to the observer). ${ }^{2}$ Furthermore, procedural spatial learning (route learning) can be distinguished from declarative spatial memory as involved in object location memory. ${ }^{3}$ In turn, recent studies found evidence for dissociations between at least two cognitive processes within object location memory: (1) exact, metric positional encoding: positional memory, and (2) binding object identity information to locations: object location binding. ${ }^{4}$ Moreover, a third possible integration mechanism might exist that combines these two processes, referred to as the combined process. ${ }^{5}$ Selective effects have been found on these three mechanisms in healthy subjects under experimental conditions. ${ }^{467}$ In line with this, neuropsychological studies have shown that dysfunctions of specific brain structures, such as the medial temporal lobe and hippocampus, ${ }^{8}$ or the parietal cortex, ${ }^{9}$ may cause selective impairments in different aspects of spatial memory. In addition, specialised involvement of these cortical areas in specific aspects of spatial processing has been shown using positron emission tomography $\left(\mathrm{PET}^{10}\right)$ and functional MRI. ${ }^{11}$

The purpose of the present study was to find evidence for selective neuropsychological impairments on either of the aforementioned three object location memory processes, which would further support the hypothesised distinction. More detailed insight into spatial memory and perception may be gained, which would contribute to a more refined diagnostic arsenal for the identification and understanding of the very incapacitating deficits in this cognitive realm. Therefore, 10 patients with cerebral lesions after tumour resection were studied using a cognitive task which is sensitive for the assessment of these separate processes. Furthermore, two neuropsychological tasks that measure procedural spatial memory were included. The results of the individual patients were compared with data from 24 healthy subjects.

\section{Patients and methods}

Patients were recruited from the Department of Neurology of the University Medical Centre, Utrecht. All patients had undergone neurosurgical removal of an intracranial tumour more than 1 year before this study. None of the patients received radiotherapy after the operation. A group of 24 healthy sub- 


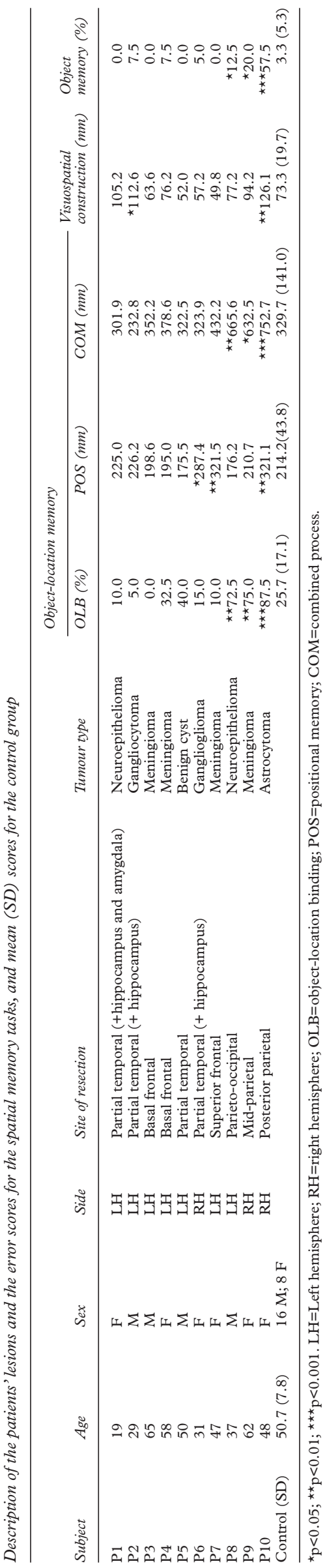

jects of comparable age and education served as controls (see the table for a detailed description of the patients and control group).

To test object location memory, all patients studied computer displays with a frame containing 10 easy to name objects at different locations, which had to be relocated afterwards. Three task conditions were used. Firstly, to measure object location binding, all different objects were shown at different locations, which had to be reassigned to their correct positions that were premarked by black dots. Secondly, to assess positional memory, objects that were all equal were shown at different locations, and had to be relocated as accurately as possible in an empty frame (without premarked dots). Thirdly, to measure the combined process, different objects were shown at different locations that had to be relocated in an empty frame (without premarked dots). ${ }^{12}$

As control tasks, conditions were included that assessed visuospatial construction and perception (the patient had to copy a frame containing 10 different objects at different locations without a memory component) and object identity memory (10 objects were shown, which had to be recognised subsequently from a set of 20 objects, containing the ones that were shown previously). Each condition consisted of two different displays. Additionally, the subject had to relocate the objects both immediately after the presentation as well as after a 3 minute delay. Performance measures were percentage incorrect items in the object location binding and object identity memory condition, and deviation ( $\mathrm{mm}$ ) in the positional memory task, the combined process, and the visuospatial construction and perception condition. Neuropsychological evaluation on the basis of single tasks may produce spurious data, as patients often fail on tasks for reasons unrelated to the specific function of interest. Therefore, combined error scores were used based on both the immediate and the delayed performance. To measure procedural spatial memory, two standard neuropsychological spatial memory tasks that are widely used in clinical practice were included, The Corsi block tapping task to determine the spatial memory span ${ }^{13}$ and the Oxford stylus maze to assess spatial learning. ${ }^{14}$ All spatial tasks can be presumed to assess allocentric spatial processes, due to the presence of external frames of reference. ${ }^{2}$ The results of the control group were used to calculate standardised $Z$ scores for the individual patients. Subsequently, cut off scores were determined for a performance that differed from the control mean at 0.05 ( $Z>1.65), 0.01(Z>2.33)$, and 0.001 $(Z>3.10)$ levels of statistical significance. ${ }^{15}$

\section{Results}

The table shows the results for the 10 patients on the experimental spatial memory and control tasks, as well as the mean scores for the control group. Five patients (P1-P5) were unimpaired on all spatial memory tasks, whereas one patient (P10) displayed impairments on all of these. Four patients were 
impaired on object location memory. Of these, two patients (P6, P7) showed a selective impairment on positional memory, and two other patients (P8, P9) performed worse than the controls on object location binding and the combined process. For the non-spatial memory control tasks, two patients (P2, P10) performed poorly on visuospatial construction and perception, and three patients (P8-P10) had impaired object identity memory. Analysis of the performance on the standard neuropsychological spatial memory tasks showed a lowered performance on the Oxford stylus maze in three patients (P6, P9, P10; p<0.001), whereas one patient was impaired on the Corsi block tapping task (P10; $\mathrm{p}<0.001)$.

For lesion localisation, it was found that the occurrence of impaired object location memory was more frequent in right hemispheric patients than in left hemispheric patients (Mann-Whitney $U=3.00, Z=1.96$, $\mathrm{p}=0.03$ ). Furthermore, object location memory dysfunction was more often found in patients with posterior (parietal or occipital) lesions than in patients with anterior (temporal or frontal) lesions (Mann-Whitney $U=3.00$, $Z=1.96, \mathrm{p}=0.03)$.

\section{Discussion}

The purpose of the current study was to find selective neuropsychological deficits in specific components within object location memory. Our results showed a double dissociation between the performance on positional memory (the ability to remember precise, metric information) on the one hand, and the object location binding and the combined task conditions on the other hand. These findings provide further evidence that object location memory consists of multiple cognitive mechanisms. It seems that one process exists (positional memory) that is responsible for the encoding and retrieval of euclidean positional information, whereas a separate process is involved in the assignment of item information to (relative or precise) spatial locations (as measured with the object location binding and combined process conditions).

No selective impairments in either the object location binding or the combined process were seen in this study (in line with previous findings $\mathrm{s}^{4}$ ), which suggests that these conditions may measure the same underlying process. Moreover, the patients that have slight problems in memory for the identity of objects (P8, P9) are also impaired at these two spatial memory conditions. However, these general memory deficits cannot fully explain the selective spatial memory findings, as it is especially spatial mnemonic processing that is tested, rather than memory for object identity (patients do not have to recall the objects). Notably, patients with deficits in object location memory did not always display impairments on maze learning or block span memory. This corroborates the distinction between memory for spatiotemporal relations (procedural learn- ing of routes or sequences) and declarative spatial memory (remembering the locations of objects). ${ }^{3}$

Interestingly, the results show that all three right hemispheric patients are impaired on the spatial memory tasks, but only one left hemispheric patient (out of seven). This is in line with the idea that spatial deficits are commonly (but not solely) the result of right hemispheric damage. ${ }^{16}$ Furthermore, all patients with posterior lesions performed poorly, regardless of side affected. This clearly shows that the posterior parietal cortices are important in spatial memory processing. ${ }^{91114}$ One patient (P6) had a lesion in the (medial) right temporal lobe (with complete removal of the right hippocampus). This brain area is also involved in object location memory. ${ }^{8}$

In summary, the results of the present study show a double dissociation between positional memory and the assignment of object identities to (relative or precise) locations. Although strong conclusions about the functional neuroanatomical circuits of spatial memory cannot be made on the basis of single cases, it seems that in this group of patients spatial memory dysfunction is especially present in those with either right hemispheric damage or posterior lesions.

We thank Dr M J B Taphoorn (Department of Neurology, University Medical Centre Utrecht) for his help in selecting the patients.

1 McCarthy RA, Evans JJ, Hodges JR. Topographic amnesia: spatial memory disorder, perceptual dysfunction, or category specific semantic memory impairment? f Neurol Neurosurg Psychiatry 1996;60:318-25.

2 Holdstock JS, Mayes AR, Cezayirli E, et al. A comparison of egocentric and allocentric spatial memory in medial temporal lobe and Korsakoff amnesics. Cortex 1999;35: 479-501.

3 Schacter DL, Nadel L. Varieties of spatial memory: a problem for cognitive neuroscience. In: Lister RG, Weingartner HJ, eds. Perspectives on cognitive neuroscience. New York: HJ, eds. Perspectives on cognitive neuroscie

4 Postma A, De Haan, EHF. What was where? Memory for object locations. Q f Exp Psychol A 1996;49:178-99.

5 Kessels RPC, Postma A, Wester AJ, et al. Memory for object locations in Korsakoff's amnesia. Cortex 2000;36:47-57.

6 Postma A, Izendoorn R, De Haan EHF. Sex differences in object location memory. Brain Cogn 1998;36:334-45.

7 Postma A, Meyer G, Tuiten A, et al. Effects of testosterone administration on selective aspects of object-location memory in healthy young women. Psychoneuroendocrinology 2000;25:563-75.

8 Milner B, Johnsrude I, Crane J. Right medial temporal-lobe contribution to object-location memory. Philos Trans $R$ Soc Lond B Biol Sci 1997;352:1469-74.

9 Stein JF. The representation of egocentric space in the posStein JF. The representation of egocentric space in the pos-
terior parietal cortex. Behav Brain Sci 1992;15:691-700.

10 Jonides J, Smith EE, Koeppe RA, et al. Spatial working memory in humans as revealed by PET. Nature 1993;363: 623-5.

11 Zarahn E, Aguirre GK, D'Esposito M. Temporal isolation of the neural correlates of spatial mnemonic processing with fMRI. Cogn Brain Res 1999;7:255-68.

12 Kessels RPC, Postma A, De Haan EHF. Object relocation: a program for setting up, running, and analyzing experiments on memory for object locations. Behav Res Methods Instrum Comput 1999;31:423-8.

13 Berch DB, Kirkorian R, Huha EM. The Corsi blocktapping task: methodological and theoretical considerations. Brain $\operatorname{Cog} n$ 1998;38:317-38.

14 Ratcliff G, Newcombe F. Spatial orientation in man: effects of left, right, and bilateral posterior cerebral lesions. $7 \mathrm{Neu}$ of left, right, and bilateral posterior cereb
rol Neurosurg Psychiatry 1973;36:448-54.

15 De Haan EHF, Heywood CA, Young AW, et al. Ettlinger revisited: the relation between agnosia and sensory impairments. F Neurol Neurosurg Psychiatry 1995;58:350-6.

16 Milner B. Interhemispheric differences in the localization of psychological processes in man. Br Med Bull 1971;27:272-7. 